\title{
Talent Rising; People Analytics \& Technology \\ driving Talent Acquisition Strategy
}

Gavin Walford-Wright MA, FCIPD

Middlesex University (Doctoral Candidate) and Oxford Strategic Consulting gavin@walford-wright.com

\section{Professor William Scott-Jackson}

University of Oxford, Cass Business School and Oxford Strategic

Consulting

wsj@oxfordstrategicconsulting.com 


\section{Introduction}

'Talent Rising; People Analytics \& Technology driving Talent Acquisition Strategy' is a study of the opportunities that have been created through technological advancement in the Talent Acquisition industry and how this links to strategic HR management (SHRM) and business strategy. It focuses on how an organisation can embrace the world's leading technology and compose a unique technology stack to overcome its challenges in talent acquisition with three core objectives; reduce time to hire (rewiring existing processes to lean process in the tech), reduce cost per hire (internal capability and technology vs. recruitment agencies) and increase quality of hire (measured by engagement, retention and performance).

Through the analysis of the research and literature review, it was found that a single source system does not exist which pulls all the best practice together in an end to end system. Therefore a unique technology stack (Figure 1: 'Talent Rising') was designed to be universally adopted and fully integrated, together with legacy systems where required:

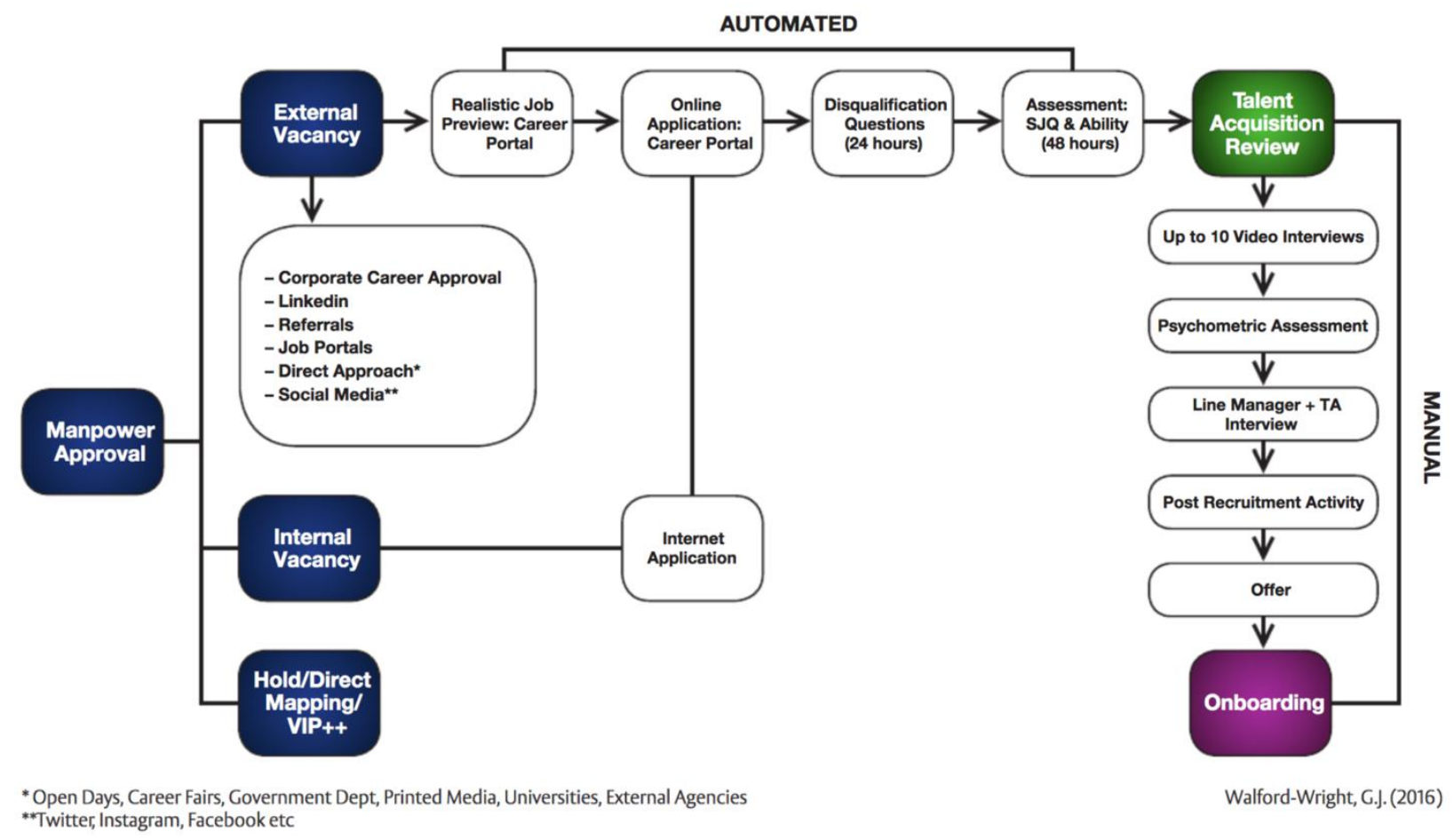

Figure 1: Talent Rising 
In 2016, Gert Leonhard said that 'humanity will change more in the next 20 years than in the previous 300.' As the world enters this period of truly transformational change driven by technological advances and people analytics, one new source of competitive advantage is candidate centricity; deeply understanding your candidates' needs and fulfilling them better than anyone else (Driest et al, 2016). To achieve this level of centricity, data is required but must be transformed into insight and strategy. This can be delivered though people analytics and technology. The world may have moved on significantly from Michaels' (et al, 2001) War for Talent, but the increasingly competitive landscape for recruiting and retaining talented employees is still a differentiator.

It is clear that in this war for talent that by 2025 the candidate pool will have shrunk. The 2015 McKinsey Global Growth Model study (covering 2005-2015) found there were three times as many workers as retirees. However, within the next decade this ratio will have fallen to $1: 1$. This coupled with the need for specialised talent, especially in the technology and life sciences fields, makes it clear that candidates are in the driver's seat, often entertaining multiple job offers. Employees can choose the organisation whose values align with their own and that lays out a clear path to career advancement for them.

There is considerable published evidence which demonstrates how heavily influenced job applicants are by the way they are treated during the selection process (Lievens and Chapman 2010: 137-40). Thus the task of reducing cost and time per hire has to be carefully balanced with the third objective of increasing the quality of hire

\section{Talent Acquisition Challenges}

Resourcing and talent management, like other HR activities, must focus on supporting the organisation in achieving its core strategic objectives However; our research suggests that the current scenario, in many organisations, is a lack of core strategic components;

- No clear vision

- No ATS (applicant tracking system)

- Low engagement

- No people analytics

- No referral program 
- High cost per hire (due to using external agencies)

- Long time to hire (due to lack of automation and manual handoffs)

- Lower quality of hire (due to no or poor assessments and hiring process)

- Poor relationships (lack of trust) with hiring managers and internal applicants

- No KPIs or IDPs (individual development plans) for talent acquisition team

The above became apparent through our meetings with Talent Acquisition teams globally and reviewing internal surveys across organisations. It was clear that a lack of technology and poor processes led to candidate and internal client dissatisfaction. Thus organisations must develop and maintain a world class talent acquisition strategy within the constraints of budgets, volatile business and labour markets and increasing competition, both commercially, and in the 'war for talent'.

\section{People Analytics}

People analytics uses data to inform management decisions, be it in talent acquisition, retention, placement, promotion, compensation, or workforce and succession planning. People analytics and big data are now must-have capabilities in HR. The promise of people analytics is that it will almost certainly move HR forward in terms of analysis and insight but will also create the ability for predictive and suggestive technology. However, as our research has shown, less than half of HR leaders believe that they link their data to key business and financial data. The possibilities in linking different data sources together to generate actionable insights is uncovering surprising sources of talent and counterintuitive insights about what drives employee performance.

As early as 2010, Davenport et al (2010) had noticed that leading companies such as Google, Best Buy, P\&G, and Sysco were using sophisticated data collection technology and analysis to get the most value from their talent. These companies had taken the guesswork out of employee management by leveraging analytics to improve their methods of attracting and retaining talent, connecting their employee data to business performance, differentiating themselves from competitors, and more.

The CIPD's latest research report on the utilisation of analytics in the region (CIPD 2015) found that organisations in the region view HR analytics as a topic of huge potential, but believe that the capability of their teams, systems and people are not 
enabling them to realise the full potential of their HR data. This is a true reflection on most organisations which are slow to transition from the Ulrich (1997) model with a focus on becoming an admin expert, to the Ulrich (2005) model of understanding and becoming a functional expert and human capital developer.

\section{Talent Technology}

Talent Technology is the point where systems and processes of human resources management and technology intersect; where the basic functions of HR meet with technology. Talent Technology makes the routine tasks of HR easier and more efficient through technology. In recent years, the automation of Talent Acquisition processes has developed beyond the traditional job boards (Monster, Indeed, LinkedIn) and into social media, outreach and applicant tracking systems. According to the CIPD's 2015 research, organisations are increasingly using technology to recruit (see Figure 2). In the same research, a quarter of organisations reported that technology was replacing some jobs within their organisations.

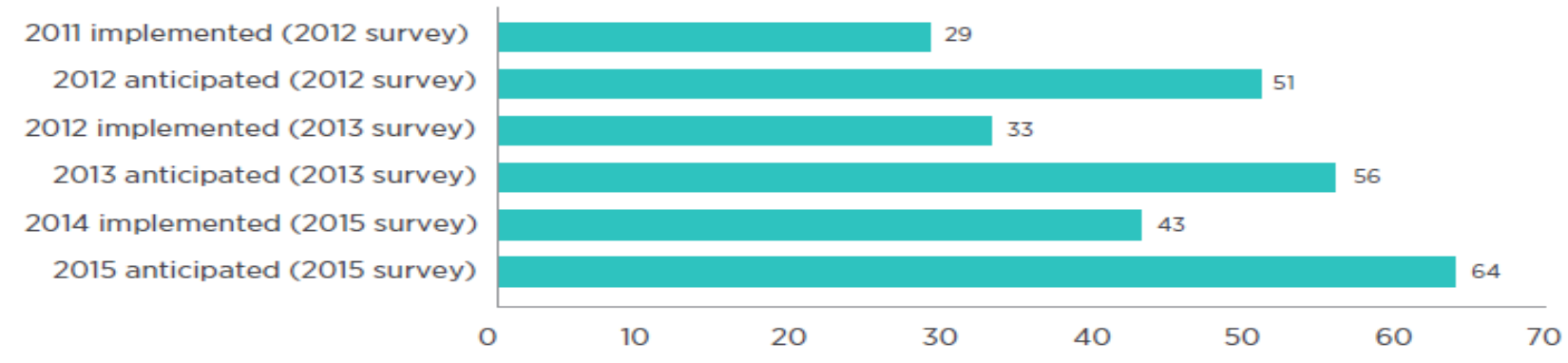

Base: 519 (2015 survey); 438 (2013 survey); 501 (2012 survey)

Figure 2: The use of Technology to Recruit (\% of respondents)

Advanced software algorithms can identify talent and match it to an organization's needs, pinpointing team players based on core traits and personality matching, making it an effective method for taking care of costly and time-consuming preliminary screening. In 2014, Gartner correctly predicted that the market for Big Data and analytics would generate US $\$ 3.7$ Trillion in products and services and generate over 4.4 million new jobs by 2015 . 


\section{Talent Acquisition Process}

Bock (2015), based on his experience as Senior Vice President of People Operations at Google, suggests that it 'doesn't matter who you are, where you're from or what school you went to'. Clearly Google has a global presence and hires people across the world, but the principal is the same; we shouldn't screen out based on human bias, but actively seek out 'non-traditional' applicants who are different to typical hires in terms of age, educational background, gender etc. It could be argued that for many roles, cognitive ability is the single most valuable assessment in the talent acquisition process.

The three classic strategies for competitive advantage in business (Porter 1985) can be linked to the talent acquisition objectives of an organisation: cost leadership (reducing the cost per hire); innovation (reducing the time to hire); and quality (improving the quality of hire).

Talent acquisition leaders are more conscientious of the candidate experience than ever before and 2018 has seen a rise in adoption of new services and investments to make a lasting and positive impression on future employees. Services have become more personalised as the likes of candidate concierge services come into play. With this approach candidates are sent a link to download an app for their mobile device that would offer GPS guidance to the exact location of an interview, detailed background info about the people they are meeting, and when they arrive on the corporate campus, geo-location beacons will send notifications as candidates pass campus landmarks. The talent acquisition team gives special tours of other departments, delivers presentations on the company culture and provides refreshments between interviews.

\section{The Use of Technology in Selection Techniques}

The benefit of using technology to assist in the selection process enables recruitment teams to focus their time and resources on the candidates with the highest potential. The process takes into account a number of key requirements for the basis of selection decisions:

1) Does the person fit with our organisation culture?

2) Do they have potential to learn quickly and grow in the new environment / role?

3) Have they got the experience and knowledge required to operate at the necessary level of independence? 
4) Do they behave in line with the required competencies for the role?

In order to evaluate the above and as a result of much literature review and research, the following selection tools were successfully implemented and utilised in several organisations by the author:

- An organisational culture fit tool for the purpose of sifting

- Cognitive ability assessments for the purpose of sifting

- Video Interviewing tools for the purpose of screening

- Personality tools for the purpose of selecting

The gold standard in talent acquisition was always the resume and a personal interview. However, anyone can write anything on a resume and face to face interviews favour those who are articulate and personable. Talent acquisition teams often had to rely on instinct (gut) in making a decision on whether a candidate moves forward in the process or is rejected. With the technology now available, the creation of new streams of verifiable information about potential hires means that, as Linkedln's VP of Talent Solutions and Insights, Dan Shapero says: "recruiting has always been an art, but it's becoming a science."

The latest talent acquisition technology enables the creation of a dialogue with candidates and takes them on an interactive journey where they learn about the organisation, through a realistic job preview, and themselves, through a psychometric assessment. There is a danger of course, that candidates with low computer skills would be discouraged and at a disadvantage through a talent acquisition process based on technology. However, as organisations are becoming more technology-based, being 'tech savvy' is a requirement for most organisations. Therefore, if an organisation can naturally discount and screen out candidates that are unable to complete the application process, then this actually adds value to the process and gives the candidates an honest experience of what working for a technology-based organisation entails.

In addition to its application during selection, talent technology and people analytics' greatest HR value is as a predictive tool. Through creating a process to analyse the skills and attributes of high performers (and low performers), people analytics allows organisations to build a 'template' for future hires. It is also critical to not just focus 
on high performers; it is as important to understand what under performance looks like so that a competency and behavioural assessment can be created to screen out those candidates that are unlikely to succeed and rank those that have the potential. The right talent technology and people analytics stack allows you to see candidates threedimensionally (3D): the candidate is brought to life and not only can you see and hear the candidate, there is an underlying factual result from the assessment the candidate has talent as part of the process. The people analytics derived from the technology is democratic, impartial, supporting of a meritocracy and enables companies to make smarter decisions. Google, for example, has an entire HR division devoted to "people analytics" which measures qualities such as social skills, flexibility, emotional intelligence, initiative, attitude (negative or positive or "good fit" vs. "bad fit"), and perseverance.

Through the use of automated, customised talent technology the talent acquisition team can cast the net wide and far and significantly expand the hiring pool. The talent acquisition team can go online to sites such as Linkedln and search the world for that perfect hire; it's no longer, about narrowing the talent pool to active candidates restricted by geography. Facebook, and Linkedln, for example, can be used to market to passive potential candidates (i.e. not looking to switch jobs).

This is good for the candidate too. If an individual is on the market for a new opportunity, having a professional and relevant online presence is a game-changing way to increase their visibility. The greater a candidate's online presence, the more chance an organisation will become aware of the candidate and their accomplishments. Referring back to my earlier comment, the goal is build a 3D online portrait of a candidate; this can include pictures, letters of praise and recommendation, slideshows, videos, and even an interactive resume. The goal is to create a win-win opportunity for the candidate and company; the organisation wants to find you and you want to be found.

\section{Online Integrated Assessment Solution}

Of course, the adoption of technology and people analytics, as part of an online integrated assessment solution, does not in itself automatically enable organisations to achieve their primary talent goals. The skills of the talent acquisition team must be developed to extract maximum benefit from the systems and understand the results of the assessments. Moreover, the basic HR goals still hold true; the goal of recruitment is to 
make successful placements and the goal of selection is to hire the most talented and diverse employees. Technology is a facilitator to achieve these goals with maximum efficiency and effectiveness; but organisation values are typically a complex combination of personal beliefs, attitudes and behaviours.

Although several authors suggest that recruitment is a personal and interactive journey; more recent research indicates that the best candidates are found when we remove the overriding human bias inherent in manual selection and evaluation. Resourcing and talent acquisition strategy takes place within an overall HR strategy and aligned fully with corporate strategy. The literature points to a talent acquisition process which is automated and unbiased. Diversity in the workplace is no longer a nice to have; it's a must. Most businesses intuitively know this, but until recently the value of a diverse and inclusive workforce had not been fully quantified. Today, there's ample research showing that having more diverse and inclusive workplace makes good business sense. Companies with diverse workforces are not only more creative and innovative, they also perform better financially. Google's Director of People Analytics, Dr. Brian Welle, explains how this works out in Google's favour; "if we have an employee base that reflects our [diverse] user base, we are going to better understand the needs of people all over the world."

\section{Final Thoughts}

Technology is no longer the preserve of IT; it is entwined throughout any organisation and it's deployment has become a mandatory skill set for the modern HR practitioner. Leading organisations are successfully using people analytics to tackle some of their most challenging HR issues, such as talent acquisition, talent pipeline planning, organisational development, engagement, and learning and talent development. The CIPD's (2015) research discovered that many organisations recognise the potential for people analytics to enable them to outperform their competitors and recognise it as a key capability to develop if they are to ensure business growth. As a result, many organisations are now investing in the IT infrastructure, HR strategies and team capabilities to be able to complete high-quality people analytics.

There is a counterbalance for this lust for technology and people analytics. This article is not necessarily an indictment of the invasive use of technology; rather, it is an 
affirmation of its efficacy in enabling progressive organisations to achieve their operational objectives. However, technology initiatives should be considered in view of the human factor that is the real driver of long-term success. People, not technology, are responsible for ensuring a steady progress toward the goal of attaining organisational excellence under the umbrella of a galvanizing vision and a robust mission. The tipping point is where futurism meets humanity and in attracting talent to an organisation; technology can make or break the talent connection. 


\section{Reference List}

1. BOCK, L. (2015) Work Rules! Twelve Books

2. BOXALL, P. \& PURCELL, J. (2003) Strategy and Human Resource Management. Palgrave Macmillan

3. CIPD (2013) Talent Analytics and Big Data: the challenge for HR. London. Chartered Institute of Personnel and Development.

4. CIPD (2015) Employee turnover and retention. London. Chartered Institute of Personnel and Development.

5. CIPD (2015) Evolution of HR analytics: A Middle East perspective. London. Chartered Institute of Personnel and Development.

6. CIPD (2015) Market Pricing \& Job Evaluation. London. Chartered Institute of Personnel and Development.

7. CIPD (2015) Resourcing and talent planning. London. Chartered Institute of Personnel and Development.

8. CIPD (2015) What will talent acquisition look like in 2016? London. Chartered Institute of Personnel and Development.

9. Corporate Leadership Council Report (2010) "Building Next Generation Recruiting Capabilities".

10. CRESWELL, J. (2007) Qualitative Inquiry and Research Design: Choosing among Five Approaches ( $2^{\text {nd }}$ Edn). Thousand Oaks, CA: Sage. 
11. DAVENPORT, T.H., HARRIS, J. and SHAPIRO, J. (2010) Competing on Talent Analytics. Harvard Business Review, October.

12. LEONHARD, G. (2016) Technology vs. Humanity. London. FutureScapes.

13. LIEVENS, F. and CHAPMAN, D. (2010) Recruitment and selection. London: Sage

14. MCKINSEY \& COMPANY (1997) 'War for Talent'

15. MICHAELS, E., HANDFIELD-JONES, H. and AXELROD, B. (2001) The War for Talent. Harvard Business Press

16. ULRICH, D. (1997). Human Resource Champions. Boston: Harvard Business School Press.

17. ULRICH, D. and BROCKBANK, W. (2005) The HR Value Proposition. Harvard Business School Publishing. 


\section{List of Figures}

Figure

Page

Figure 1: Talent Rising

2

Figure 2: The use of Technology to Recruit (\% of respondents)

13 I P a g e Gavin Walford-Wright \& William Scott-Jackson. Strategic HR Review. Talent Rising. 